\title{
Malignant Postcricoid Neoplasm
}

National Cancer Institute

\section{Source}

National Cancer Institute. Malignant Postcricoid Neoplasm. NCI Thesaurus. Code C9323.

A primary or metastatic malignant neoplasm that affects the postcricoid region. 